\title{
Nuclear spirals: Gas in asymmetric galactic potential with a massive black hole
}

\author{
Witold Maciejewski ${ }^{1}$ \\ ${ }^{1}$ Obserwatorium Astronomiczne Uniwersytetu Jagiellońskiego, Orla 171, 30-244 Kraków, \\ Poland
}

\begin{abstract}
Nuclear spirals can provide a wealth of information about the nuclear potential in disc galaxies. They form naturally as a gas response to non-axisymmetry in the gravitational potential, even if the degree of this asymmetry is very small. Linear wave theory well describes weak nuclear spirals, but stronger asymmetries in the potential induce waves beyond the linear regime, which appear as spiral shocks. If a central massive black hole (MBH) is present, spiral shocks can extend all the way to its immediate vicinity, and generate gas inflow up to $0.03 \mathrm{M}_{\odot} \mathrm{yr}^{-1}$. This coincides with the accretion rates needed to power local Active Galactic Nuclei.
\end{abstract}

\section{Introduction}

Recent high-resolution maps of galactic centres (Regan \& Mulchaey 1999, Martini \& Pogge 1999, Pogge \& Martini 2002, Martini et al. 2003 a,b) indicate that $50 \%$ to $80 \%$ of disc galaxies possess nuclear spirals in their innermost hundreds of parsecs. It has been proposed that nuclear spirals may be related to the fueling of Seyfert activity (Regan \& Mulchaey 1999). Martini \& Pogge (1999) showed that nuclear spirals are not selfgravitating, and that they are likely to be shocks in nuclear gas discs.

Here I investigate the dynamics of nuclear spirals which are density waves generated in gas by a rotating potential. In particular, I am interested in how the gas flow in the nucleus is modified by the presence of a central massive black hole $(\mathrm{MBH})$ or a density cusp. I construct hydrodynamical models of nuclear spirals with the code that follows the gas flow on a polar grid in a fixed asymmetric potential of a weak oval or of a strong bar. Potentials with a $10^{8} \mathrm{M}_{\odot}$ massive black hole $(\mathrm{MBH})$ or without a $\mathrm{MBH}$ (e.g. constant-density core) are considered here. Self-gravity in gas is not taken into account, and I assume isothermal equation of state for 'hot gas' (sound speed $c_{S}=20 \mathrm{~km} \mathrm{~s}^{-1}$ ). In order to minimize spurious inflow resulting from the inner boundary on the polar grid, I impose the reflection boundary condition there. Full account of this work will be published elsewhere (Maciejewski 2004 a,b).

\section{Zones of propagation for nuclear spirals}

Nuclear spirals can be treated as waves in gas (Goldreich \& Tremaine 1979, Englmaier \& Shlosman 2000, Maciejewski 2004a), and in the tight-winding limit they follow the dispersion relation for wavenumber $k,\left(\Omega+\kappa / m-\Omega_{B}\right)\left(\Omega-\kappa / m-\Omega_{B}\right)=k^{2} c_{S}^{2} / m^{2}$, when excited by a tumbling $m$-fold pattern of frequency $\Omega_{B}=$ const. In the formula above, $\Omega$ is the angular velocity in the disk, and $\kappa$ the epicyclic frequency. Note that for a twofold pattern (e.g. a bar) in the galaxy centre $\Omega+\kappa / 2-\Omega_{B}$ is always positive, therefore $\Omega-\kappa / 2-\Omega_{B}$ must be positive in order for the spiral wave to propagate. Therefore the presence of nuclear spirals in galaxies can tell us about the potential in the galaxy centre: since $\Omega_{B}=$ const, then if the nuclear spiral extends to the galaxy centre, $\Omega-\kappa / 2$ must be 

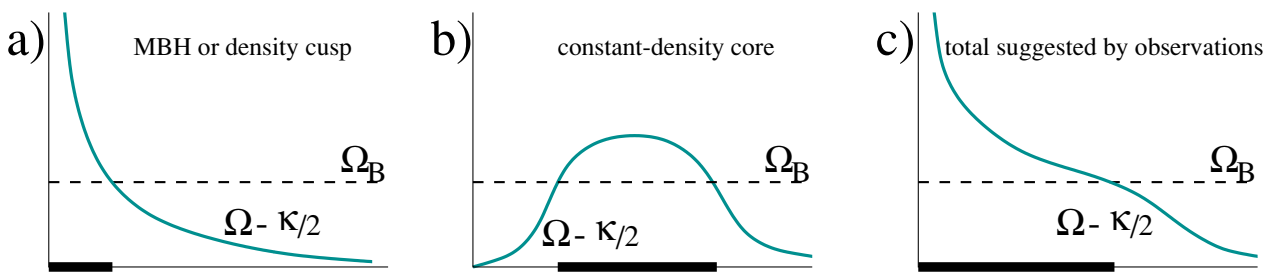

Figure 1. Typical frequency curves (frequency as a function of radius) for gravitational potentials of: a) a $\mathrm{MBH}$ or a central density cusp, b) continuous mass distribution with a constant-density core, c) a typical composite supported by common appearance of nuclear spirals. Regions where nuclear spirals can propagate are indicated by thick lines on the horizontal axis.

large there, which is characteristic for a $\mathrm{MBH}$ or a central density cusp (Fig. 1). To the contrary, constant-density core (linearly rising inner rotation curve) implies $\Omega-\kappa / 2 \equiv 0$, and therefore prohibits propagation of any nuclear spiral wave.

\section{Nuclear spirals in a weak oval}

In order to check how weak departures from axisymmetry in the stellar gravitational potential are sufficient to create nuclear spirals in gas, I built models for the potential, whose departure from axisymmetry is 10 times smaller than that for a strong bar studied by Maciejewski et al. (2002). This potential contains a weak oval distortion rather than a bar, with the maximum ratio of tangential to radial force of $2 \%$. As can be seen in Fig. 2, such a weak asymmetry is sufficient to generate a nuclear spiral with the arm/inter-arm density contrast $\sim 2$. However, velocity perturbations in the spiral are small, therefore it is a wave, not a shock, in gas. As explained by the linear theory, the nuclear spiral extends inwards from the outer inner Lindblad resonance (ILR) to the inner ILR, or to the galaxy centre in the absence of this last one (Fig. 2, middle columns). Note that despite the assumed relatively high velocity dispersion in gas $\left(c_{S}=20 \mathrm{~km} \mathrm{~s}^{-1}\right)$, to which the pitch angle of the spiral is proportional, the nuclear spiral in Fig. 2 appears tightly wound.

Although the nuclear spiral generated by a weak oval is a strong perturbation in gas density, no inflow is triggered at any radial scale (Fig. 2, bottom-right). Only at the innermost radii an episodic drop of $\sim 10^{5} \mathrm{M}_{\odot}$ occurs during formation of the spiral. This corresponds to the inflow of $\sim 10^{-3} \mathrm{M}_{\odot} \mathrm{yr}^{-1}$, and may be only relevant to the feeding of weakest AGN. However, it may gain importance if nuclear spirals re-appear as a response to drivers like passing Globular Clusters or Giant Molecular Clouds.

\section{Nuclear spirals in a strong bar}

Nuclear spiral in a strong bar (here I use the potential from Maciejewski et al. 2002) propagates inwards from the inner ends of straight shocks in the bar (Fig. 3). Contrary to the spiral in a weak oval, it appears as a slight density enhancement, but with large negative $d i v^{2} \mathbf{v}$, hence it is a spiral shock. Its strength equals that of the principal straight shock in the bar, and the departures from circular velocity in the spiral are large (Fig. 3, middle-top). Pitch angle of the spiral shock is consistently larger than what the linear theory predicts (Fig. 3, middle-bottom). Its morphology does not change with time, but the density in the spiral increases, since the gas transported inwards by the straight principal shock in the bar accumulates in the nuclear spiral. 

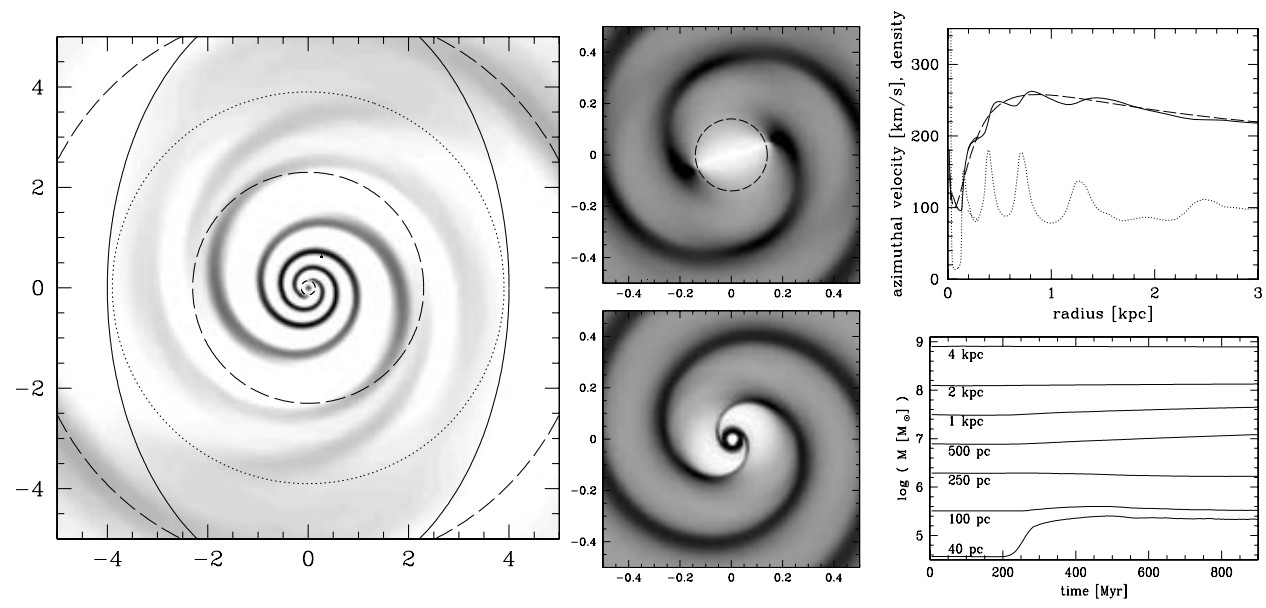

Figure 2. Left: Snapshot of gas density in a model with a weak oval asymmetry in the gravitational potential once the morphology of the flow has stabilized. Darker color indicates larger densities. The solid ellipse outlines the oval, and the dashed circles mark the outer ILR at $2.3 \mathrm{kpc}$ and the corotation at $5.6 \mathrm{kpc}$. Top-central: Zoom into the central kiloparsec of the model with a constant-density core and without a MBH. The dashed circle marks the inner ILR.

Bottom-central: Zoom into the central kiloparsec of the model with a MBH. The inner grid boundary is at the radius of $20 \mathrm{pc}$. Top-right: Azimuthal velocity (solid line) plotted against the unperturbed rotation curve (dashed line) as a function of radius, and the radial density profile (dotted line). Bottom-right: Mass accumulated within various radii as a function of time.

This accumulation of gas in the nuclear spiral is responsible for secular inflow in the spiral shock. Its timescale is much longer than that in the straight principal shock in the bar, but with time its amplitude reaches that in the straight shock: $0.7 \mathrm{M}_{\odot} \mathrm{yr}^{-1}$ (Fig. 3, right panels). At the radius of $500 \mathrm{pc}$ it happens $\sim 0.4$ Gyr after formation of the bar $(2.5$ rotations of the bar). The same large inflow will likely occur at smaller radii, but after still longer times, and evolutionary changes in the galaxy may override its importance. Nevertheless, in addition to this secular inflow, another form of inflow takes place around a $\mathrm{MBH}$ if it is present in the galaxy centre. This inflow reaches $0.03 \mathrm{M}_{\odot} \mathrm{yr}^{-1}$ after $0.5 \mathrm{Gyr}$, comparable to what is needed to sustain activity of local Seyfert galaxies (Peterson et al. 1997).

\section{Confronting observations}

Models presented here can be related to the morphological classification of the nuclei, and the statistics of nuclear activity in a recent sample of active and inactive galaxies (Martini et al. 2003 a,b). In the data, nuclear spirals classified as 'grand design' and 'loosely wound' occur in $60 \%$ of active galaxies and in $20 \%$ of inactive ones. 'Grand design' class directly corresponds to the spiral shock in the strong bar modeled here, and is not observed in unbarred galaxies. 'Loosely wound' class indicates large pitch angle of the spirals, which models associate with the spiral shock. The observational fact that both these classes occur more often in active than in non-active galaxies supports the idea that the spiral shock causes gas inflow large enough to feed the central MBH. Nuclear spirals in the 'tightly wound' class of Martini et al. tend to avoid galaxies classified as 

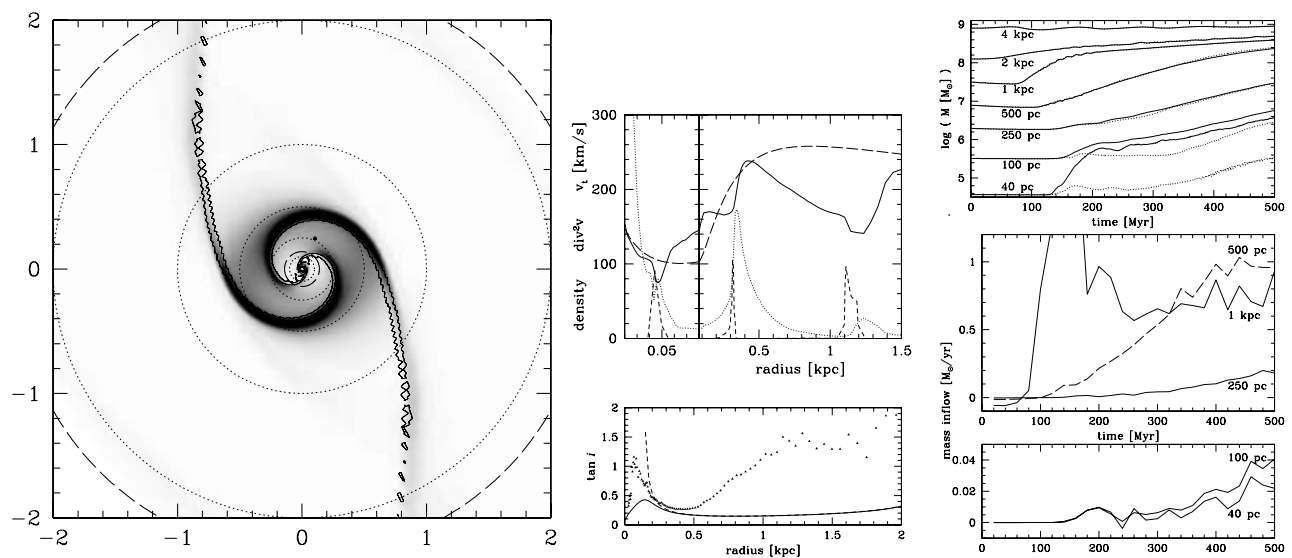

Figure 3. Left: Snapshot of gas density (greyscale), and of $\operatorname{div}^{2} \mathbf{v}$ (for $\operatorname{div} \mathbf{v}<0$, contours) in a model with a MBH and a strong bar, once the morphology of the flow has stabilized. Overplotted are dotted circles at radii where the inflow has been measured. Top-central: Azimuthal velocity (solid line) with the rotation curve (long-dashed line), radial density profile (dotted line), and $d i v^{2} \mathbf{v}$ (short-dashed line). The plot has two adjacent parts drawn in different radial scales: from $20 \mathrm{pc}$ to $80 \mathrm{pc}$, and from $80 \mathrm{pc}$ to $1.5 \mathrm{kpc}$. Bottom-central: Tangens of the pitch angle of the shock. Filled triangles mark the values measured in the hydrodynamical model, while lines are the linear prediction for a potential with a central MBH (solid), and without it (dashed). Top-right: Mass accumulated within various radii as a function of time for model with a MBH (solid line) and without it (dotted line). Bottom-right: Mass inflow as a function of time in model with a $\mathrm{MBH}$. Note the small, but not negligible inflow triggered at the innermost radii after the arrival of the spiral shock there (at about $130 \mathrm{Myr}$ ).

barred. In the models, the nuclear spiral in a weak oval can follow the linear theory for longer than in a strong bar, and therefore it can appear as tightly wound there.

\section{Conclusions}

Nuclear spirals are easily generated even by small asymmetries in the galactic potential, but they cannot form in constant-density cores. Some nuclear spirals can feed the central $\mathrm{MBH}$, but observations of gas kinematics are essential in testing it.

\section{References}

Englmaieri, P., \& Shlosman, I. 2000, ApJ, 528, 677

Goldreich, P., \& Tremaine, S. 1979, ApJ, 233, 857

Maciejewski, W. 2004a, MNRAS, in press

Maciejewski, W. 2004b, MNRAS, in press

Maciejewski, W., Teuben, P. J., Sparke, L. S., \& Stone, J. M. 2002, MNRAS, 329, 502

Martini, P., \& Pogge, R. W. 1999, AJ, 118, 2646

Martini, P., \& Regan, M. W., Mulchaey, J. S., \& Pogge, R. W. 2003a, ApJS, 146, 353

Martini, P., Regan, M. W., Mulchaey, J. S., \& Pogge, R. W. 2003b, ApJ, 589, 774

Peterson, B. M. 1997, An Introduction to Active Galactic Nuclei (Cambridge: Cambridge Univ. Press)

Pogge, R. W., \& Martini, P. 2002, ApJ, 569, 624

Regan, M. W., \& Mulchaey, J. S. 1999, AJ, 117, 2676 